\title{
Anestesia com cetamina, midazolam e óxido nitroso em cães submetidos à esofagoplastia cervical
}

\author{
Ketamine, midazolam and nitrous oxide anesthesia in dogs submitted to \\ cervical esophagoplasty
}

\section{Juliana Tabarelli Brondani ${ }^{1}$ Cláudio Corrêa Natalini ${ }^{2} \quad$ Ney Luis Pippi $^{3}$ Alexandre Mazzanti ${ }^{4}$ Luciana Prati ${ }^{5}$ Adriana Paula Bertin ${ }^{6}$}

\section{RESUMO}

Este estudo foi realizado para avaliar a anestesia intravenosa com cetamina e midazolam (K-M) em cães ventilados mecanicamente com $66 \%$ de óxido nitroso e $33 \%$ de oxigênio ou $100 \%$ de oxigênio. Foram utilizados 16 cães sem raça definida, hígidos, com peso médio de 14,2 \pm $3,78 \mathrm{~kg}$, submetidos a jejum sólido de 12 horas prévio ao procedimento. A anestesia foi induzida com a associação de cetamina $\left(10 \mathrm{mg} . \mathrm{kg}^{-1}\right)$ e midazolam $\left(0,5 \mathrm{mg} \cdot \mathrm{kg}^{-1}\right)$ administrados na mesma seringa por via intravenosa (IV). Para manutenção anestésica, foi utilizada cetamina (5mg. $\left.\mathrm{kg}^{-1}\right)$ e midazolam $\left(0,25 \mathrm{mg} \cdot \mathrm{kg}^{-1}\right)$ administrados por via IV em intervalos de 10 minutos. Os animais foram distribuidos em dois grupos: $\mathrm{N}_{2} \mathrm{O}_{\text {e }} \mathrm{O}_{2}$. $\mathrm{No}$ grupo $\mathrm{N}_{2} \mathrm{O}$, os cães foram ventilados mecanicamente com $66 \%$ de óxido nitroso e 33\% de oxigênio. No grupo $\mathrm{O}_{2}$, somente o oxigenio foi utilizado para ventilação artificial. Em ambos os grupos, os animais foram submetidos à esofagoplastia cervical. As variáveis fisiológicas utilizadas para comparação entre os grupos foram: freqüencia cardiaca, pressões arteriais sistólica, média e diastólica, saturação de oxigênio da hemoglobina e temperatura corporal. A necessidade ou não de doses adicionais da associação cetamina e midazolam também foi registrada para comparação. A análise estatística dos resultados não demonstrou diferenças significativas nas variáveis fisiológicas entre os grupos. No grupo $O_{2}$, foram necessárias doses maiores da associação $K-M$ para manutenção anestésica nos 30 minutos iniciais $(p<0,05)$. Conclui-se que a anestesia injetável com $K-M$, nas doses utilizadas neste estudo, com ou sem inclusão de óxido nitroso, não deprime a função cardiovascular, não causa hipotermia, promove indução tranqüila e recuperação satisfatória com salivação e produz adequado grau de analgesia e relaxamento muscular para realização de cirurgias no esôfago cervical em cães. A inclusão do óxido nitroso permite a utilização de doses menores da associação cetamina-midazolam.

Palavras-chave: cetamina, midazolam, óxido nitroso, cães.

\section{ABSTRACT}

This study was conducted to evaluate the effects of ketamine, midazolam, and nitrous oxide anesthesia ( $K-M)$ in dogs artificially ventilated with $66 \%$ nitrous oxide and $33 \%$ oxygen or $100 \%$ oxygen. These dogs were submitted to experimental cervical esophagoplasty. Sixteen clinically healtly mixed breed dogs with mean body weight of $14.2 \pm$ $3.78 \mathrm{~kg}$ were studied. A 12 -hour fasting period was established for each dog. Anesthesia was produced with $10 \mathrm{mg} . \mathrm{kg}^{-1} \quad$ ketamine and $0.5 \mathrm{mg} . \mathrm{kg}^{-1} \quad$ midazolam administered intravenously. Anesthesia was maintained with $5 \mathrm{mg} . \mathrm{kg}^{-1}$ and $0.25 \mathrm{mg} . \mathrm{kg}^{-1}$ midazolam IV as a bolus injection every 10 minutes after induction. Two groups were studied: $\mathrm{N}_{2} \mathrm{O}$ and $\mathrm{O}_{2}$. In one group $\left(\mathrm{N}_{2} \mathrm{O}\right) 8$ dogs were artificially ventilated with $66 \%$ nitrous oxide $\left(\mathrm{N}_{2} \mathrm{O}\right)$ and $33 \%$ oxygen

${ }^{1}$ Médico Veterinário, Mestrando do Programa de Pós-graduação em Medicina Veterinária, Universidade Federal de Santa Maria (UFSM). Hospital Veterinário, Faixa de Camobi, Km 9, 97105-900, Santa Maria, RS. E-mail: jtbrondani@yahoo.com Autor para correspondência.

${ }^{2}$ Médico Veterinário, Professor Veterinary Anesthesiology and Pain Management, PhD, Louisiana State University - Baton Rouge - EUA.

${ }^{3}$ Médico Veterinário, Professor Adjunto, PhD, UFSM.

${ }^{4}$ Médico Veterinário, Mestre, Doutor, UFSM.

${ }^{5}$ Médico Veterinário, Mestre Cirurgia Veterinária, UFSM.

${ }^{6}$ Acadêmica do Curso de Medicina Veterinária, UFSM. 
$\left(\mathrm{O}_{2}\right)$. In the other group $\left(\mathrm{O}_{2}\right) 8$ dogs were artificially ventilated with $100 \% \mathrm{O}$. Both groups were submitted to an experimental cervical ésophagoplasty. In order to compare both treatments heart rate, systolic, diastolic, and mean blood pressure, pulse oximetry and body temperature were recorded. Analgesia was compared between groups as the need of supplementary doses of $K-M$. The results demonstrated that $K-M$ injectable anesthesia do not produce cardiovascular depressant effects and hypothermia, promotes quiet induction and satisfactory recovery with salivation and produces adequate analgesia and muscular relaxation to cervical esophagus in dogs. Additional doses of $K-M$ were necessary $(p<0.05)$ in the $\mathrm{O}_{2}$ group suggesting that nitrous oxide decreases the dose of $K-M$ in dogs.

Key words: ketamine, midazolam, nitrous oxide, dogs.

\section{INTRODUÇÃO}

Os anestésicos inalatórios voláteis causam depressão cardiovascular dose-dependente, seja por depressão direta do miocárdio, ou diminuição da atividade simpática (STEFFEY, 1996). Quando se deseja manutenção da estabilidade cardiovascular, uma alternativa aos anestésicos voláteis é o uso de técnicas de anestesia injetável balanceada. Segundo HELLYER et al. (1991), as associações cetamina-midazolam ou cetamina-diazepam não afetam significativamente o sistema cardiovascular e respiratório. JACOBSON \& HARTSFIELD (1993) também constataram que a associação cetamina-midazolam induz mínimos efeitos cardiorrespiratórios.

A cetamina, por produzir hipertonia muscular, recuperação disfórica e convulsões, tem sido utilizada em associação com sedativos ou tranqüilizantes, que eliminam ou minimizam esses efeitos excitatórios (HELLYER et al., 1991). Os benzodiazepínicos como diazepam e midazolam são utilizados associados à cetamina. O midazolam é hidrossolúvel e, conseqüentemente, compatível com a cetamina em solução (JACOBSON \& HARTSFIELD, 1993), além de ser três vezes mais potente que o diazepam (HELLYER et al., 1991). Benzodiazepínicos lipossolúveis como o diazepam podem causar precipitação da solução quando misturados com cetamina (BROWN et al., 1993). Para potencializar o efeito analgésico da associação cetamina-midazolam/ diazepam, foram utilizados óxido nitroso em suínos (LESSER et al., 1993) ou opióides como butorfanol ou oximorfona em cães (JACOBSON et al., 1994). A utilização de óxido nitroso e cetamina-midazolam ainda não foi descrita em cães.

O objetivo deste estudo foi avaliar os efeitos cardiovasculares da anestesia com cetamina- midazolam e óxido nitroso e a viabilidade do seu uso para cirurgia no esôfago cervical em cães.

\section{MATERIAL E METODOS}

Foram utilizados 16 cães adultos, 10 fêmeas e 6 machos, sem raça definida, com peso médio de $14,2 \pm 3,78 \mathrm{~kg}$, provenientes do Biotério Central da UFSM. Os animais foram considerados hígidos após exame clínico completo e nas 12 horas anteriores ao procedimento os cães foram submetidos a jejum sólido prévio ao procedimento anestésico-cirúrgico.

Os cães foram distribuídos aleatoriamente em dois grupos $\left(\mathrm{O}_{2}\right.$ e $\left.\mathrm{N}_{2} \mathrm{O}\right)$ de 8 animais. Em ambos os tratamentos, a indução foi realizada com cetamina ${ }^{a}$ na dose de $10 \mathrm{mg} . \mathrm{kg}^{-1}$ e midazolam ${ }^{\mathrm{b}}$ na dose de $0,5 \mathrm{mg} \cdot \mathrm{kg}^{-1}$, administrados na mesma seringa, através de injeção intravenosa em "bolus". A manutenção anestésica foi realizada com metade da dose de indução de cetamina e midazolam (5mg.kg-1 e 0,25 mg. $\mathrm{kg}^{-1}$ respectivamente), em intervalos regulares de 10 minutos, via IV. Doses adicionais da associação K-M para manutenção do plano anestésico foram administradas quando necessário, e registradas para variável de comparação entre os grupos. Para a administração dessas doses adicionais, usaram-se os seguintes parâmetros: aumento do tono mandibular, aumento do reflexo palpebral, aumento de 30 a 40\% da freqüência cardíaca e pressão arterial média em relação ao valor basal e perda do relaxamento muscular na área operatória (informada pelo cirurgião). Aos vinte minutos anteriores ao término da cirurgia, cessava-se a administração dos agentes intravenosos (cetamina-midazolam) e seguia-se apenas a ventilação mecânica com $\mathrm{O}_{2}$ ou com $\mathrm{O}_{2}$ e $\mathrm{N}_{2} \mathrm{O}$ até 10 minutos anteriores ao final do procedimento.

No grupo $\mathrm{O}_{2}$, os cães foram ventilados mecanicamente com $100 \%$ de oxigênio, e no grupo $\mathrm{N}_{2} \mathrm{O}$, com $66 \%$ de óxido nitroso e $33 \%$ de oxigênio. O ventilador foi ajustado a um volume corrente de 20 a $30 \mathrm{ml}^{\mathrm{kg}} \mathrm{kg}^{-1}$, com pressão de admissão de 12 a $15 \mathrm{~cm}$ de $\mathrm{H}_{2} \mathrm{O}$ e freqüência respiratória de 12 a 16 ciclos por minuto. $\mathrm{O}$ óxido nitroso foi interrompido 10 minutos antes do término da administração de $\mathrm{O}_{2}$ A veia cefálica foi cateterizada percutamente com cateter no 20, para administração dos agentes anestésicos e solução cloreto de sódio 0,9 \% na dose de $10 \mathrm{ml} . \mathrm{kg} \cdot \mathrm{h}^{-1}$. Todos os animais foram submetidos à esofagoplastia cervical experimental para implantação de um segmento de diafragma homólogo.

Foram avaliadas as seguintes variáveis: freqüência cardíaca (oxímetro de pulso ${ }^{\mathrm{d}}$ ); pressões arteriais sistólica, média e diastólica (método oscilométrico $^{\mathrm{e}}$ não invasivo), saturação de oxigênio 
da hemoglobina (oximetria de pulso ${ }^{\mathrm{d}}$ ) e temperatura retal (termometria eletrônica ${ }^{\mathrm{f}}$ ). A temperatura da sala cirúrgica foi mantida ao redor de $25^{\circ} \mathrm{C}$. Foi mantida uma temperatura ambiente constante, com o intuito de minimizar o máximo o efeito dessa sobre a temperatura dos animais. Doses adicionais da associação cetamina-midazolam para manutenção do plano anestésico foram registradas e comparadas entre os grupos. As variáveis fisiológicas foram mensuradas anteriormente à indução e em intervalos de 10 minutos após indução até o final da cirurgia.

A qualidade da indução e recuperação anestésica e o grau de relaxamento muscular trans e pós-cirúrgico imediato (30 minutos após término da cirurgia) foram observados. A qualidade da indução e recuperação foram avaliadas utilizando os parâmetros explicados a seguir. A indução foi considerada boa quando ocorreu uma tranqüila transição para inconsciência, livre de sinais de excitação; satisfatória quando estavam presente movimentos de pedalagem (leve ataxia) e vocalização; e pobre quando, além desses havia tentativa de fuga e defecação. Apenas foi observado o pós-operatório imediato (30 minutos após término da cirurgia) e a recuperação foi considerada boa quando livre de agitação, pedalagem e vocalização; satisfatória quando pedalagem (ataxia) e vocalização estavam presentes e pobre quando além de pedalagem (ataxia) e vocalização observavam-se também muita agitação e defecação. O grau de relaxamento muscular foi avaliado pelo tono mandibular e, subjetivamente, pelo cirurgião, que informava se havia adequado relaxamento muscular na área operatória, necessário para realização do procedimento cirúrgico.

Os resultados obtidos foram comparados estatisticamente através da análise de variância (ANOVA), e comparação de médias pelo teste de Bonferroni. O grau de significância estabelecido foi de $5 \%(\mathrm{p}<0,05)$.

\section{RESULTADOS}

As variáveis freqüência cardíaca (FC), saturação de oxigênio da hemoglobina $\left(\mathrm{SPO}_{2}\right)$, pressões arteriais sistólica (PAS), média (PAM) e diastólica (PAD) e temperatura retal $(\mathrm{T})$, não diferiram estatisticamente entre os tratamentos e se mantiveram dentro da faixa de variação fisiológica para cães (Tabela 1).

A freqüência cardíaca, no grupo $\mathrm{O}_{2}$, aumentou significativamente em relação ao valor basal em todos os tempos de avaliação, exceto T30 (Tabela 1). A temperatura corporal reduziu-se significativamente no grupo $\mathrm{N}_{2} \mathrm{O}$ nos tempos T40 a
T70 em relação ao valor basal, porém, dentro da faixa de variação fisiológica para cães (Tabela 1).

Doses adicionais da associação K-M foram necessárias para manutenção anestésica no grupo $\mathrm{O}_{2}$ Aos 20 minutos após indução foram necessárias doses estatisticamente maiores $(\mathrm{p}<0,05)$ de cetamina $(8,75$ $\pm 0,528)$ e midazolam $(0,44 \pm 0,026)$ no grupo $\mathrm{O}_{2}$ em relação as doses de cetamina $(5,0 \pm 0,528)$ e midazolam $(0,25 \pm 0,026)$ do grupo $\mathrm{N}_{2} \mathrm{O}$.

A indução anestésica foi boa em todos os animais, tranqüila, sem sinais de vocalização ou pedalagem. O grau de relaxamento muscular para realização do procedimento cirúrgico foi considerado adequado, pelo cirurgião, em ambos os grupos, e ocorreu redução do tono muscular mandibular durante todo período anestésico-cirúrgico. A recuperação anestésica foi considerada satisfatória, pois os animais apresentaram ataxia (movimentos pedalagem) e salivação.

\section{DISCUSSÃO}

A cetamina é considerada um fármaco que aumenta a tonicidade simpática, com elevação significativa da freqüência cardíaca, débito cardíaco, pressão arterial sistêmica, trabalho do ventrículo esquerdo e consumo de oxigênio pelo miocárdio (LIN, 1996). Entretanto, a administração de diazepam anteriormente à cetamina minimiza esse efeito estimulante cardiovascular (HASKINS et al., 1986).

No presente estudo, no grupo $\mathrm{O}_{2}$, a cetamina está associada somente ao midazolam. Ainda assim, observam-se aumentos significativos $(\mathrm{p}>0,05)$ na freqüência cardíaca. A explicação mais razoável para aumento da freqüência cardíaca com o uso de cetamina é o aumento do tono simpático (JACOBSON \& HARTSFIELD, 1993). No grupo $\mathrm{N}_{2} \mathrm{O}$, observa-se que este efeito estimulante da cetamina sobre o sistema cardiovascular está minimizado, pois não há aumento significativo da freqüência cardíaca nesse grupo. Segundo LIN (1996), o efeito estimulante da cetamina sobre o sistema cardiovascular é minimizado ou inibido pela administração anterior ou concomitante de fármacos como benzodiazepínicos, fenotiazínicos, agonistas-alfa $\mathrm{a}_{2}$ e anestésicos inalatórios, incluindo óxido nitroso.

No presente estudo, os efeitos estimulantes da cetamina sobre o sistema cardiovascular, provavelmente, foram minimizados pelo uso concomitante de midazolam ou midazolam e óxido nitroso. A associação cetamina-midazolam, neste trabalho, não provocou depressão da função cardiovascular o que pode ser evidenciado pelos 
Tabela 1 - Valores médios e erro padrão da média da freqüência cardíaca (FC - batimentos/minuto), pressão arterial (mmHg) sistólica (PAS), média (PAM) e diastólica (PAD), saturação de oxigênio da hemoglobina $\left(\mathrm{SPO}_{2}-\%\right)$, temperatura corporal $\left(\mathrm{T}^{\circ} \mathrm{C}\right)$, cetamina $(\mathrm{mg} / \mathrm{kg})$ e midazolam $(\mathrm{mg} / \mathrm{kg})$ nos grupos $\mathrm{O}_{2}$ e $\mathrm{N}_{2} \mathrm{O}$ nos diversos tempos de avaliação.

\begin{tabular}{|c|c|c|c|c|c|c|c|c|c|}
\hline \multirow{2}{*}{ Variáveis } & \multirow{2}{*}{ Grupos } & \multicolumn{8}{|c|}{ Tempo (min) } \\
\hline & & 0 & 10 & 20 & 30 & 40 & 50 & 60 & 70 \\
\hline \multirow[t]{2}{*}{$\mathrm{FC}(\mathrm{bpm})$} & $\mathrm{O}_{2}$ & $97,8 \pm 9,22$ & $140,0 \pm 9,22^{*}$ & $144,4 \pm 9,22^{*}$ & $130,6 \pm 9,22$ & $137,3 \pm 9,22^{*}$ & $137,0 \pm 9,22^{*}$ & $143,4 \pm 9,22^{*}$ & $147,5 \pm 9,22^{*}$ \\
\hline & $\mathrm{N}_{2} \mathrm{O}$ & $90,4 \pm 9,22$ & $127,8 \pm 9,22^{*}$ & $119,8 \pm 9,22$ & $115,5 \pm 9,22$ & $109,8 \pm 9,22$ & $115,0 \pm 9,22$ & $121,6 \pm 9,22$ & $120,8 \pm 9,22$ \\
\hline \multirow[t]{2}{*}{ PAS (mmHg) } & $\mathrm{O}_{2}$ & $130,4 \pm 8,73$ & $129,1 \pm 8,73$ & $148,1 \pm 8,73$ & $163,3 \pm 8,73$ & $147,5 \pm 8,73$ & $147,9 \pm 8,73$ & $149,5 \pm 8,73$ & $149,3 \pm 8,73$ \\
\hline & $\mathrm{N}_{2} \mathrm{O}$ & $137,1 \pm 8,73$ & $116,0 \pm 8,73$ & $114,8 \pm 8,73$ & $141,5 \pm 8,73$ & $127,4 \pm 8,73$ & $135,5 \pm 8,73$ & $138,9 \pm 8,73$ & $136,6 \pm 8,73$ \\
\hline \multirow[t]{2}{*}{ PAM (mmHg) } & $\mathrm{O}_{2}$ & $96,0 \pm 5,64$ & $110,2 \pm 5,64$ & $93,0 \pm 5,64$ & $133,0 \pm 5,64^{*}$ & $97,6 \pm 5,64$ & $98,8 \pm 5,64$ & $112,2 \pm 5,64$ & $101,2 \pm 5,64$ \\
\hline & $\mathrm{N}_{2} \mathrm{O}$ & $98,8 \pm 5,64$ & $91,2 \pm 5,64$ & $97,0 \pm 5,64$ & $116,2 \pm 5,64$ & $110,4 \pm 5,64$ & $100,8 \pm 5,64$ & $111,0 \pm 5,64$ & $104,4 \pm 5,64$ \\
\hline \multirow[t]{2}{*}{$\mathrm{PAD}(\mathrm{mmHg})$} & $\mathrm{O}_{2}$ & $82,6 \pm 5,08$ & $78,0 \pm 5,08$ & $85,4 \pm 5,08$ & $82,8 \pm 5,08$ & $91,2 \pm 5,08$ & $89,0 \pm 5,08$ & $91,6 \pm 5,08$ & $89,8 \pm 5,08$ \\
\hline & $\mathrm{N}_{2} \mathrm{O}$ & $85,2 \pm 5,08$ & $85,4 \pm 5,08$ & $90,2 \pm 5,08$ & $96,6 \pm 5,08$ & $95,2 \pm 5,08$ & $92,6 \pm 5,08$ & $91,0 \pm 5,08$ & $86,8 \pm 5,08$ \\
\hline \multirow[t]{2}{*}{ SPO2 (\%) } & $\mathrm{O}_{2}$ & $96,7 \pm 0,937$ & $97,1 \pm 0,937$ & $97,7 \pm 0,937$ & $98,2 \pm 0,988$ & $97,9 \pm 0,937$ & $96,3 \pm 0,988$ & $97,8 \pm 0,927$ & $98,1 \pm 0,927$ \\
\hline & $\mathrm{N}_{2} \mathrm{O}$ & $94,8 \pm 1,052$ & $95,9 \pm 0,885$ & $97,0 \pm 0,931$ & $97,2 \pm 0,981$ & $97,2 \pm 1,052$ & $96,3 \pm 0,931$ & $97,6 \pm 0,982$ & $96,0 \pm 0,885$ \\
\hline \multirow[t]{2}{*}{$\operatorname{TEMP}\left({ }^{\circ} \mathrm{C}\right)$} & $\mathrm{O}_{2}$ & $38,9 \pm 0,371$ & $38,5 \pm 0,371$ & $38,5 \pm 0,371$ & $38,7 \pm 0,371$ & $38,8 \pm 0,371$ & $38,9 \pm 0,371$ & $39,0 \pm 0,371$ & $38,9 \pm 0,371$ \\
\hline & $\mathrm{N}_{2} \mathrm{O}$ & $39,0 \pm 0,371$ & $38,8 \pm 0,371$ & $38,7 \pm 0,371$ & $38,5 \pm 0,371$ & $38,3 \pm 0,371^{*}$ & $38,3 \pm 0,371^{*}$ & $38,2 \pm 0,371^{*}$ & $38,1 \pm 0,371^{*}$ \\
\hline \multirow[t]{2}{*}{$\begin{array}{l}\text { Cetamina } \\
(\mathrm{mg} / \mathrm{kg})\end{array}$} & $\mathrm{O}_{2}$ & $10,0 \pm 0,528$ & $6,25 \pm 0,528$ & $8,75 \pm 0,528^{\#}$ & $5,0 \pm 0,528$ & $5,63 \pm 0,528$ & $3,13 \pm 0,528$ & $0,63 \pm 0,528$ & $0,00 \pm 0,000$ \\
\hline & $\mathrm{N}_{2} \mathrm{O}$ & $10,0 \pm 0,528$ & $5,0 \pm 0,528$ & $5,0 \pm 0,528^{\#}$ & $5,0 \pm 0,528$ & $5,0 \pm 0,528$ & $3,13 \pm 0,528$ & $1,25 \pm 0,528$ & $0,00 \pm 0,000$ \\
\hline \multirow{2}{*}{$\begin{array}{l}\text { Midazolam } \\
(\mathrm{mg} / \mathrm{kg})\end{array}$} & $\mathrm{O}_{2}$ & $0,50 \pm 0,026$ & $0,31 \pm 0,026$ & $0,44 \pm 0,026^{\#}$ & $0,25 \pm 0,026$ & $0,28 \pm 0,026$ & $0,16 \pm 0,026$ & $0,03 \pm 0,026$ & $0,00 \pm 0,000$ \\
\hline & $\mathrm{N}_{2} \mathrm{O}$ & $0,50 \pm 0,026$ & $0,25 \pm 0,026$ & $0,25 \pm 0,026^{\#}$ & $0,25 \pm 0,026$ & $0,25 \pm 0,026$ & $0,16 \pm 0,026$ & $0,06 \pm 0,026$ & $0,00 \pm 0,000$ \\
\hline
\end{tabular}

* Diferença estatisticamente significativa $(\mathrm{p}<0,05)$ em relação ao valor basal $(\mathrm{T} 0)$.

\# Diferença estatisticamente significativa $(\mathrm{p}<0,05)$ entre grupos.

valores de pressão arterial e freqüência cardíaca obtidos, o que também foi observado por HELLYER et al. (1991), JACOBSON \& HARTSFIELD (1993), e por LESSER et al. (1993), quando o óxido nitroso foi adicionado a cetamina-diazepam em suínos.

A utilização de doses menores da associação K-M no grupo $\mathrm{N}_{2} \mathrm{O}$, quando comparadas ao grupo $\mathrm{O}_{2}$, sugere analgesia adicional promovida pelo óxido nitroso. STEFFEY (1996) e PADDLEFORD (1999) recomendam o uso do óxido nitroso como anestésico adjuvante a outros agentes inalatórios ou injetáveis. Segundo STEFFEY (1996), embora o óxido nitroso não apresente potência para produzir anestesia, quando associado a agentes inalatórios voláteis, ele aumenta a velocidade de indução e diminui a concentração alveolar necessária do agente volátil para manutenção anestésica, por um efeito carreador do óxido nitroso, devido a sua baixa solubilidade sangüínea. $\mathrm{O}$ mecanismo pelo qual o óxido nitroso produz analgesia, quando associado a agentes injetáveis, ainda não está elucidado, embora STEFFEY (1996) comente sobre o efeito depressor do sistema nervoso central (SNC). Segundo FINCK et al. (1995), a analgesia promovida pelo óxido nitroso envolveria a liberação de opióides endógenos derivado das pro-encefalinas.

A indução anestésica tranqüila desprovida de excitação e vocalização, o adequado relaxamento muscular e a recuperação anestésica satisfatória com salivação obtidos neste experimento com o uso da associação cetamina-midazolam, concordam com os achados de HELLYER et al. (1991) e JACOBSON \& HARTSFIELD (1993). O uso da cetamina isolada produz anestesia com hipertonicidade muscular e excessivos movimentos espontâneos, indução e recuperação violenta e convulsões (HELLYER et al., 1991). Os efeitos excitatórios da cetamina sobre o SNC são decorrentes da dissociação córtico-talâmica 
causando rigidez muscular e efeitos estimulatórios do sistema límbico (HASKINS et al., 1985; LIN, 1996).

$\mathrm{O}$ efeito deletério excitatório da cetamina é eliminado ou minimizado pela administração conjunta de sedativos ou tranqüilizantes. Os derivados benzodiazepínicos, como o midazolam, reduzem os efeitos excitatórios da cetamina pois promovem relaxamento muscular e hipnose (HELLYER et al., 1991).

O principal mecanismo de ação dos benzodiazepínicos é através da estimulação de receptores do ácido g-amino butírico (GABA), considerado o principal neurotransmissor inibitório do sistema nervoso central de mamíferos (CORTOPASSI \& FANTONI, 2002).

O óxido nitroso não produz relaxamento muscular (STOELTING, 1999), conseqüentemente, não era esperado que houvesse adicional relaxamento muscular no grupo $\mathrm{N}_{2} \mathrm{O}$ quando comparado com o grupo $\mathrm{O}_{2}$. Já foi relatado o desenvolvimento, em humanos, de rigidez da musculatura esquelética com uso de óxido nitroso (HORNBEIN et al., 1982).

HELLYER et al. (1991) observaram diminuição significativa na temperatura corporal, caracterizando hipotermia, com a associação cetaminamidazolam e cetamina-diazepam, enquanto HASKINS et al. (1985) observaram aumento da temperatura corporal, atribuído à atividade muscular, quando a cetamina é utilizada isoladamente. Neste estudo, a temperatura corporal manteve-se dentro da faixa de variação fisiológica para espécie.

\section{CONCLUSÃO}

A anestesia injetável com a associação cetamina (10mg.kg-1 indução e $5 \mathrm{mg} . \mathrm{kg}^{-1}$ manutenção) e midazolam $\left(0,5 \mathrm{mg} \cdot \mathrm{kg}^{-1} \mathrm{I}\right.$ e $\left.0,25 \mathrm{mg} \cdot \mathrm{kg}^{-1} \mathrm{M}\right)$, em intervalos regulares de 10 minutos, via intravenosa, com ou sem inclusão de óxido nitroso, não deprime a função cardiovascular, não causa hipotermia, promove indução tranqüila e recuperação satisfatória, com salivação, produz adequado grau de analgesia, e relaxamento muscular para realização de procedimentos cirúrgicos no esôfago cervical de cães. A inclusão do óxido nitroso permite a utilização de doses menores da associação cetamina-midazolam.

\section{FONTES DE AQUISIÇÃO}

a - Dopalen (100 mg/ml) - Agribrands do Brasil Ltda. - Divisão Saúde Animal - Paulínia, SP - Brasil.

b - Dormire $(15 \mathrm{mg} / 3 \mathrm{ml})$ - Cristália - Produtos Químicos Farmacêuticos Ltda. - Itapira, SP - Brasil.

c - NARCO-FLOW II-A - Narcosul Aparelhos Científicos Ltda.
- Porto Alegre, RS - Brasil.

d / f - Monitor Multiparamétrico configurado TEB MPC 10. e - Monitor de Pressão Arterial Oscilométrico. Critikon. Dinamap 845 XT. USA.

\section{REFERÊNCIAS BIBLIOGRÁFICAS}

BROWN, S.A.; JACOBSON, J.D.; HARTSFIELD, S.M. Pharmacokinetics of midazolam administered concurrently with ketamine after intravenous bolus or infusion in dogs. Journal of Veterinary Pharmacology and Therapeutics. v.16, n.4, p.419425, 1993.

CORTOPASSI, S.R.G.; FANTONI, D.T. Medicação préanestésica. In: Anestesia em cães e gatos. São Paulo : Roca, 2002. Cap.13, p.151-158.

FINCK, A.D.; SAMANIEGO, E.; NGAI, S.H. Nitrous oxide selectively releases Met5-enkephalin and Met5-enkephalin-Arg6Phe7 into canine third ventricular cerebrospinal fluid. Anesthesia and Analgesia, v.80, n.4, p.664-670, 1995.

HASKINS, S.C.; FARVER, T.M.; PATZ, J.D. Ketamine in dogs. American Journal of Veterinary Research, v.46, p.1855-1860, 1985.

HASKINS, S.C.; FARVER, T.M.; PATZ, J.D. Cardiovascular changes in dogs given diazepam and diazepam-ketamine. American Journal of Veterinary Research, v.47, p.795-798, 1986

HELLYER, P.W.; FREEMAN, L.C.; HUBBELL, J.A. Induction of anesthesia with diazepam-ketamine and midazolam-ketamine in greyhounds. Veterinary Surgery, v.20, n.2, p.143-147, 1991 .

HORNBEIN, T.F. et al. The minimum alveolar concentration of nitrous oxide in man. Anesthesia Analgesia, v.61, p.553 -556, 1982.

JACOBSON, J.D.; HARTSFIELD, S.M. Cardiovascular effects of intravenous bolus administration and infusion of ketamine-midazolam in dogs. American Journal of Veterinary Research, v.54, n.10, p.1710-1714, 1993.

JACOBSON, J.D.; MCGRATH, C.J.; SMITH, E.P. Cardiorespiratory effects of induction and maintenance of anesthesia with ketamine-midazolam combination, with ou without prior administration of butorphanol ou oxymorphone. American Journal of Veterinary Research, v.55, n.4, p.543$550,1994$.

LESSER, T.; EBNER, E.; ZWIENER, U. Ketaminediazepam N O combination anesthesia - a new "circulatorysystem-sparing" type of anesthetic in experimental surgery. Research in Experimental Medicine, v.193, n.4, p.207211,1993 .

LIN, H.C. Dissociative anesthetics. In: LUMB, W.V.; JONES, E.W. Veterinary anesthesia. 3. ed. Baltimore : Williams \& Wilkins, 1996. Cap.10, p.241-296.

PADDLEFORD, R.R. Anesthetic agents. In: Manual of small animal anesthesia. Philadelphia : Saunders, 1999. Cap.3, p.31-77.

Ciência Rural, v. 33, n. 6, nov-dez, 2003. 
STEFFEY, E.P. Inhalation anesthetics. In: LUMB, W.V.; JONES, E.W. Veterinary anesthesia. 3. ed. Baltimore : Williams \& Wilkins, 1996. Cap.11, p.297-329.
StOelting, R.K. Inhaled anesthetics. In: Pharmacology and physiology in anesthetic practice. 3. ed. Philadelphia: Lippincott - Raven, 1999. Cap.2, p.36-76.

Ciência Rural, v. 33, n. 6, nov-dez, 2003. 